\title{
Role of aquaporins during teleost gametogenesis and early embryogenesis
}

\section{François Chauvigné, Cinta Zapater and Joan Cerdà*}

Laboratory of Institut de Recerca i Tecnologia Agroalimentàries, Institut de Ciències del Mar, Consejo Superior de Investigaciones Cientificas, Barcelona, Spain

\section{Edited by:}

Steffen Madsen, University of

Southern Denmark, Denmark

\section{Reviewed by:}

Guy Charmantier, Universite

Montpellier 2, France

Kenichi Ishibashi, Meiji

Pharmaceutical University, Japan

*Correspondence:

Joan Cerdà, Laboratory of Institut de Recerca i Tecnologia

Agroalimentàries, Institut de Ciències

del Mar, Consejo Superior de

Investigaciones Científicas, Passeig

maritim 37-49, 08003 Barcelona,

Spain.

e-mail: joan.cerda@irta.cat
Aquaporins are believed to be involved in homeosmotic mechanisms of marine teleosts. Increasing data suggest that these molecular water channels play critical roles associated with the adaptation of gametes and early embryos to the external spawning environment. In this mini-review, we discuss recent studies suggesting the function of aquaporin-mediated fluid homeostasis during spermatozoa activation and egg formation in teleosts. In addition, we address the potential role of water channels in osmosensing and cell migration during early embryonic development.

\section{Keywords: teleost, aquaporin, oocyte, spermatogenesis, spermatozoa, embryo}

\section{INTRODUCTION}

Marine and freshwater teleosts are constantly exposed to opposite osmotic gradients leading respectively to passive influx or efflux of water and ions. Such gains and losses are compensated through the coordinated control of osmoregulatory organs that mediate opposite fluxes within body fluids in order to maintain homeosmotic balance (Marshall and Grosell, 2006). Amongst other mechanisms, water channels or aquaporins have been implicated in this process. Recent studies have advanced this notion by revealing that teleosts harbor a large repertoire of water-selective aquaporins and water and solute (glycerol, urea) permeable aquaporins, also known as aquaglyceroporins (Cerdà and Finn, 2010; Tingaud-Sequeira et al., 2010). An overview of the evolution and nomenclature of piscine aquaporins, including the recently renamed aquaporin-1 paralogs, as well as their permeability properties and expression profiles, is provided by Cerdà and Finn (2010) and Finn and Cerdà (2011).

In this mini-review, we focus on the early stages of teleost development. Oviparous marine and catadromous teleosts release their gametes into the marine environment where external fertilization takes place. Considering that the gametes lack the adult organs that deal with ion and water balance, it seems likely that molecular adaptations should be present in the reproductive organs, gametes, and early embryos to cope with the external osmotic challenges. We therefore discuss recent studies that highlight the mechanistic roles of aquaporins, and how such channels may be associated with the adaptation of teleosts to diverse life histories and habitats.

\section{AOUAPORIN EXPRESSION AND FUNCTION DURING SPERMATOGENESIS}

During spermatogenesis diploid spermatogonia associated with somatic Sertoli cells enter meiosis to become primary and secondary spermatocytes, and eventually haploid spermatids, which elongate and differentiate into flagellated spermatozoa. In teleosts, this process is regulated by pituitary gonadotropins, via specific receptors in Leydig and Sertoli cells, through the synthesis and release of sex steroids (androgens and progestins) and growth factors, respectively (Schulz et al., 2010). The final stage of spermatogenesis or "spermiation" principally involves the production of hydrated seminal fluid that facilitates the acquisition of motility and the passage of spermatozoa through the sperm ducts (Scott et al., 2010).

In mammals, different aquaporins are found in spermatozoa, as well as in testicular germ and somatic cells (Yeung, 2010). Aquaporin-7 (AQP7) is localized in late spermatids, both in the cytoplasm and later in the plasma membrane, whereas AQP8 shows a more variable distribution from restriction to certain spermatogenic cell types to all germ cells. Elongating spermatids also express the intracellular AQP11 in the caudal cytoplasm. These three aquaporin isoforms are subsequently found in spermatozoa, as well as AQP3, although they are differentially localized along the sperm tail (Yeung, 2010; Chen et al., 2011a). Although a role of AQP8 in volume regulation of murine spermatozoa has recently been suggested (Yeung et al., 2009), knockout mice models for AQP7 and -8, have failed to show clear phenotypes during spermatogenesis or sperm viability (Yang et al., 2005; Sohara et al., 2007). The function of AQP11 is also yet uncovered because $A Q P 11$ knockout mice die of renal failure before puberty (Morishita et al., 2005). However, a recent study shows that $A Q P 3$-deficient sperm displays defects in volume regulation and excessive cell swelling upon physiological hypotonic stress in the female reproductive tract (Chen et al., 2011a).

In teleosts, as suggested for mammals, aquaporins may be involved in the hydration of the seminal fluid as well as in sperm 
physiology. However, although mRNAs of different aquaporin paralogs have been found in the teleost testis (Cerdà and Finn, 2010), their specific cellular localization and the function of the protein products during spermatogenesis remain to be investigated. Recently, Zilli et al. (2009) have investigated the role of aquaporins during the activation of spermatozoa motility in marine teleosts. In these species, the hyperosmotic challenge faced by the spermatozoa when discharged into seawater leads to a rapid water efflux. This rapid efflux, which causes membrane hyperpolarization resulting in the activation of cell motility (Alavi and Cosson, 2006; Zilli et al., 2008), was suggested to be mediated by aquaporins (Cosson et al., 2008). By using specific antibodies, Zilli et al. (2009) showed that both the water-selective Aqplaa (Raldúa et al., 2008) and the aquaglyceroporin channel Aqp10b (formerly named GLP; Santos et al., 2004) are indeed expressed in the head and flagellum of gilthead sea bream (Sparus aurata) spermatozoa. Further functional expression and sperm activation assays in the presence of mercury chloride, a non-selective inhibitor of aquaporin permeability, suggested that Aqplaa might mediate sperm activation (Zilli et al., 2009). The model proposed by the authors suggests that the hyperosmotic stimulus upon release of spermatozoa into seawater induces the accumulation of Aqplaa in the plasma membrane to facilitate the rapid water efflux. This results in the reduction of cell volume and concomitant rise of the intracellular ion concentration, which in turn activates the cAMP signaling-pathway leading to the downstream phosphorylation of the flagellar proteins and the initiation of sperm motility. This model may be premature, however, since it is based on sperm activation assays in the presence of the aquaporin inhibitor $\mathrm{HgCl}_{2}$ and the reducing compound $\beta$-mercaptoethanol (which can not reverse the mercurial inhibition of Aqp10b expressed in Xenopus laevis oocytes; Santos et al., 2004; Zilli et al., 2009), and therefore further studies using more specific inhibitors are needed. In addition, a number of key questions remain to be addressed, such as the role of Aqp10b-mediated water and/or solute transport in spermatozoa and of other aquaporins during germ cell differentiation, and the distribution and function of aquaporins in Sertoli and Leydig cells.

Whether the expression of aquaporins during teleost spermatogenesis is hormonally controlled still needs to be clarified. In mammals, AQP8 is expressed in the Sertoli cells of all testicular tubules, whereas AQP0 is only expressed within Sertoli cells in tubules containing elongating spermatids just before being released into the lumen (Hermo et al., 2004). These observations suggest that AQP0 might be hormonally regulated. However, whether AQP0 or -8 in Sertoli cells are modulated by steroids, as it occurs for AQP1 and -9 in the epididymis (Oliveira et al., 2005) or for AQP2 in the uterus (Jablonski et al., 2003), remains to be investigated. In teleosts, the hydration of the seminal fluid during spermiation and acquisition of sperm motility seems to be regulated by progestins such as 17,20 $\beta$-dihydroxypregn-4-en-3-one $(17,20 \beta \mathrm{P})$ or

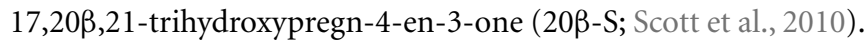
These steroids can potentially activate nuclear progestin receptors expressed in Sertoli and Leydig cells, spermatogonia and/or spermatocytes (Miura et al., 2006; Chen et al., 2010b, 2011b; Hanna et al., 2010). In addition, progestins can directly stimulate spermatozoa hypermotility through membrane receptors coupled to adenylyl cyclase located in the plasma membrane (Tubbs et al., 2011). Therefore, it will be of interest to investigate if testicular and sperm aquaporins may be under $17,20 \beta \mathrm{P}$ - or $20 \beta$-S-mediated transcriptional and/or posttranslational regulation.

\section{ROLE AND MOLECULAR REGULATION OF AQUAPORINS IN THE 00CYTE}

In mammalian oocytes, expression of mRNAs encoding different aquaporins has been described (e.g., Edashige et al., 2000), although so far detection of the corresponding polypeptides remained elusive, except for AQP3 in bovine oocytes (Jin et al., 2011). In the granulosa and theca cells associated to the oocyte, however, several functional aquaporins such as AQP1-4, -5, and -9 are differentially localized (Skowronski et al., 2009; Thoroddsen et al., 2011).

As an adaptation to the hyperosmotic condition of seawater, the oocytes of oviparous marine teleosts hydrate during meiosis resumption (oocyte maturation). This mechanism provides a water reservoir in the embryo to compensate for the passive water efflux until osmoregulatory organs develop, and improves oxygen exchange and egg dispersal in the ocean (Fyhn et al., 1999; Finn and Kristoffersen, 2007; Cerdà, 2009; Finn and Fyhn, 2010). Early studies in the gilthead sea bream (Fabra et al., 2005, 2006), and later in the Japanese eel (Anguilla japonica; Kagawa et al., 2009, 2011), identified the novel role of Aqplab in this process. This duplicated paralog facilitates the temporal water permeation and the preovulatory swelling of the oocyte. The mechanism is coregulated with yolk proteolysis and ion fluxes that generate the intracellular osmotic driving force for fluid transport, a feature well established in both old and modern teleost species (Cerdà et al., 2007; Kristoffersen et al., 2009). Further functional, genomic and phylogenetic analyses revealed that Aqplab belongs to a teleost-specific subfamily of water-selective aquaporins, which evolved by tandem duplication of a common ancestor (Martinez et al., 2005; Raldúa et al., 2008; Tingaud-Sequeira et al., 2008, 2010; Zapater et al., 2011). Interestingly, $a q p 1 a b$ transcripts have also been found in the ovary of the freshwater teleost stinging catfish (Heteropneustes fossilis), in which oocytes moderately hydrate during meiotic maturation (Singh and Joy, 2010), although the role of Aqp1ab in the oocyte of this species is yet unknown (Chaube et al., 2011).

The role of Aqp lab during oocyte hydration in marine teleosts is supported experimentally by the observation that the swelling of oocytes is blocked by aquaporin inhibitors such as mercury and tetraethylammonium (Fabra et al., 2005, 2006; Kagawa et al., 2009). However, these compounds can also affect $\mathrm{K}^{+}$channels and other ion transport proteins (Armstrong, 1990; Jacoby et al., 1999), which may play a role for inorganic osmolyte accumulation in the oocyte (Cerdà et al., 2007; Kristoffersen and Finn, 2008). More conclusive data have been recently obtained in the Atlantic halibut (Hippoglossus hippoglossus), a marine teleost that reproduces at low temperature and spawns one of the largest pelagic eggs known (Zapater et al., 2011). In this study, Atlantic halibut oocytes undergoing hydration were microinjected with an antiserum specific for halibut Aqp1ab, resulting in a dose-dependant inhibition of oocyte hydration in the presence of yolk hydrolysis. The immunological inhibition could be fully reversed by the artificial expression of halibut Aqplaa which is not recognized by 
the antibody. Therefore, these findings indicate that the decrease of oocyte hydration of Atlantic halibut oocytes can be directly related to the loss of function of Aqplab, providing for the first time functional evidence of the essential physiological role of this water channel.

Recent studies in the gilthead sea bream have begun to dissect the molecular mechanisms involved in the physiological regulation of Aqp1b in the oocyte. Preliminary data suggest that transcriptional activation of the $a q p 1 a b$ promoter in primary growth oocytes may be dependent on Sry-related high mobility group [HMG]-box (sox) genes, as well as on the nuclear progestin receptor, which are highly expressed in oogonia (Zapater et al., unpublished data). This mechanism likely results in the accumulation of high levels of $a q p 1 a b$ transcripts and Aqplab peptides in primary growth oocytes. Subsequently, Aqplab-containing vesicles are transported toward the oocyte cortex throughout the period of oocyte growth, and during meiotic maturation and hydration they are temporarily inserted into the oocyte plasma membrane (Fabra et al., 2006). Structural analyses have revealed that the cytoplasmic tail of Aqp1ab, although highly divergent among teleosts, retains specific motifs that regulate vesicular trafficking, and therefore they may be involved in the control of Aqp 1ab translocation into the oocyte plasma membrane during hydration (TingaudSequeira et al., 2008; Chaube et al., 2011). These processes appear to involve alternative mechanisms of phosphorylation and/or dephosphorylation of specific C-terminal residues, but the specific intracellular signaling pathways involved are yet unknown. These observations thus indicate that Aqp 1ab in the oocyte is tightly regulated at the transcriptional and post-translational level during oogenesis, oocyte growth, and meiotic maturation.

The studies carried out so far are revealing that the Aqplabmediated mechanism of oocyte hydration in marine teleosts is a conserved and highly regulated process, based on the interplay between osmolyte generation and the controlled synthesis and insertion of Aqplab at the oocyte surface. However, there are still many unresolved issues, such as the transductional pathways activated in the oocyte during hormone-induced meiotic maturation and hydration that coordinate osmolyte generation, Aqplab intracellular trafficking and meiosis resumption. In this regard, it will be of interest to investigate the role of $G$ proteincoupled progestin receptors on the oocyte surface, as well as the classical nuclear progestin receptor, during the control of Aqp 1ab trafficking, as these receptors may be the physiological transducers of progestins to activate meiosis resumption (Thomas et al., 2004). However, it is known that in the teleost ovary, in addition to $a q p 1 a b, \mathrm{mRNAs}$ encoding many other aquaporin paralogs are found (Cerdà and Finn, 2010), although the cellular sites of expression have not yet been defined. Whether the accumulation of these transcripts corresponds to maternal messengers stored in oocytes required for early development, or reflect the coordinated role of different aquaporins in ovarian fluid homeostasis, remains to be investigated.

\section{AQUAPORINS IN EMBRYONIC DEVELOPMENT}

Very limited information is available on the localization and function of aquaporins during mammalian and teleost early embryonic development. In the mammalian morula, AQP3, -8 , and -9 are detected in the cell-cell contact domains of blastomeres, despite observations that these embryos show very low water and solute permeability (Barcroft et al., 2003; Edashige et al., 2007), although this may differ among species (Jin et al., 2011). At the blastula stage, blastoderm cells express only AQP3, whereas AQP3 and -8 are accumulated at the basolateral membranes of trophectodermal epithelial cells of blastocysts, which also express AQP9 at the apical membrane (Barcroft et al., 2003). The change in the subcellular localization of these aquaporins coincides with the enhanced water permeability of blastula stage embryos, and consequently it was suggested that these channels may mediate transepithelial water and solute movements. (Barcroft et al., 2003). The function of these aquaporins could compensate each other because $A Q P 3$ knockout mice can develop to term (Ma et al., 2000). Moreover, solute permeability is not completely abolished in embryos in which AQP3 expression has been suppressed by injection of AQP3 double-stranded RNA (Edashige et al., 2007).

During teleost embryonic development, changes in the expression and cellular localization of aquaporins have only been investigated in zebrafish (Danio rerio) and common mummichog (Fundulus heteroclitus; Tingaud-Sequeira et al., 2009; Chen et al., 2010a). In zebrafish, aqp $1 a a,-3 a,-7$, and $-10 b$ transcripts are detected at the 2-4 cell and morula stages (Figure 1A), suggesting that these aquaporins are maternally inherited, as reported for mummichog aqp1aa (Tingaud-Sequeira et al., 2009). Interestingly, transcripts encoding zebrafish $a q p 3 b$ and mummichog aqp3a are first noted at the onset of gastrulation, during which they are apparently accumulated in embryos (Tingaud-Sequeira et al., 2009; Figure 1A). Later in development, aquaporin expression is generally enhanced where the expression of other paralogs such as $a q p 0 a,-0 b,-8 a a,-10 a$, and $-11 b$ may be detected. This latter expression appears to be associated with organogenesis and tissue differentiation. For example, Aqplaa and -8aa have been related to somitogenesis and vascular development (Sumanas et al., 2005; Tingaud-Sequeira et al., 2009; Chen et al., 2010a), Aqp0a and $-0 \mathrm{~b}$ to normal lens development and transparency (Froger et al., 2010), and Aqp11b to tail differentiation (Ikeda et al., 2011).

In the early embryos of common mummichog (TingaudSequeira et al., 2009) and zebrafish (Figures 1B-E), immunolocalization experiments using specific antibodies have respectively revealed the presence of Aqp3a and -3b in the blastoderm cells as well as in the enveloping layer (EVL). In the mummichog, further accumulation of the Aqp3a protein is evident in the basolateral membrane of the EVL epithelium as well as in the membrane of migrating blastomeres at the marginal region of the blastoderm (Tingaud-Sequeira et al., 2009). The localization of Aqp3a in the EVL of teleost embryos may indicate a role of this channel in water and solute transport during epiboly. Interestingly, the embryos of the common mummichog respond to environmental desiccation by accelerating development (Tingaud-Sequeira et al., 2009). Under these conditions Aqp3a expression in the EVL is reduced, which could be a mechanism to reduce evaporative water loss through the EVL, while membrane localization of Aqp3a in migrating blastomeres remains unchanged (TingaudSequeira et al., 2009). In recent years, water and solute transport 

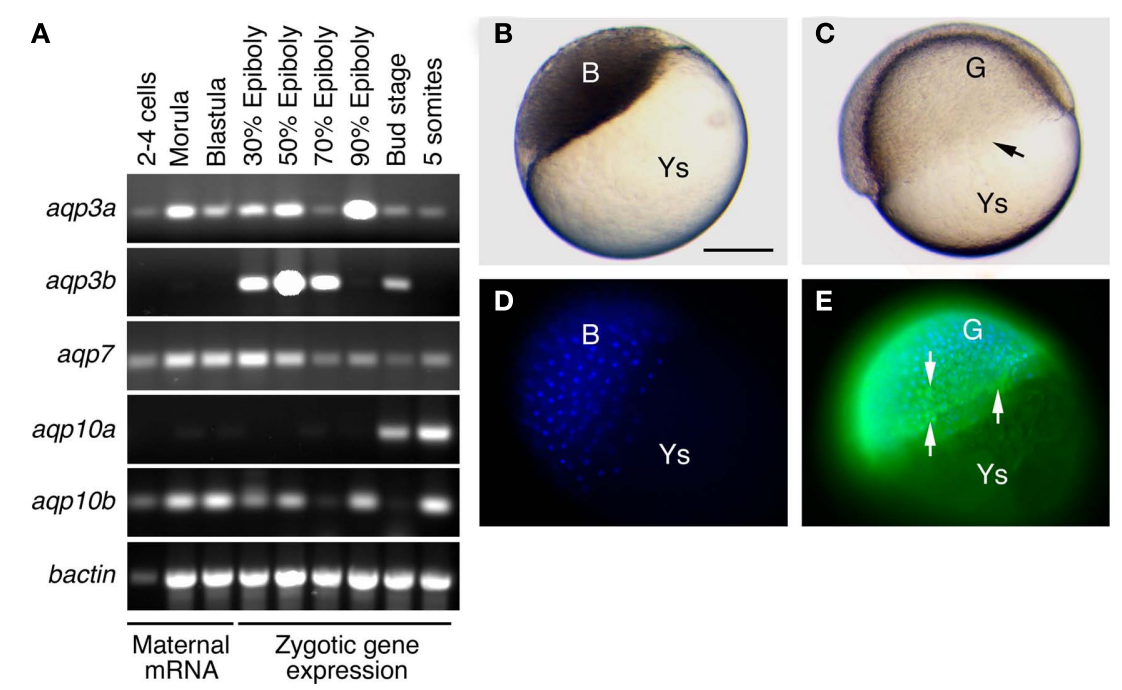

FIGURE 1 | Expression of aquaporins during teleost embryonic development. (A) Standart RT-PCR analyses of aquaporin transcriptional expression in zebrafish embryos reveals that aqp3a, -7 , and $-10 b$ transcripts are detectable throughout development, whereas aqp3b and aqp10a expression is only detected during gastrulation and onward, and at the onset of organogenesis, respectively. (B-E) Whole-mount immulocalization of
Aqp3b in zebrafish blastula embryos (left) and 50\% epiboly embryos (right) using specific antibodies (Chauvigné et al., 2011). (C) The involuting edge of the gastrula is indicated with an arrow. (D-E) Aqp3b is not detected in the blastula (D), whereas it appears in migrating blastomeres [(E) arrows] during gastrulation. Nuclei of blastomeres are counterstained with DAPI. Bar, $500 \mu \mathrm{m}$. Ys, yolk sac; B, blastomeres; G, gastrula. Bar, $500 \mu \mathrm{m}$. mediated by mammalian AQP1, -3 , or -4 has been proposed to be involved in cell migration and proliferation by driving water influx, thus facilitating lamellipodia extension and cell migration (Papadopoulos et al., 2008; Monzani et al., 2009). A similar role for AQP3 has been proposed during neural tube closure in X. laevis embryos (Cornish et al., 2009). In teleost embryos, the specific pattern of Aqp3a expression in the plasma membrane of migrating blastomeres, and its persistence in mummichog embryos showing an accelerated rate of epiboly under desiccation conditions, also suggests the involvement of this aquaporin in cell migration during gastrulation. Preliminary experiments in common mummichog have shown delayed epiboly of embryos injected with an specific antibody against Aqp3a, and thus these observations may support the role of this aquaporin during cell migration (Chauvigné and Cerdà, unpublished data). This potentially conserved role requires however further investigation. Moreover, teleost embryos also express other aquaporins such as aqp $1 a a$, 7, and $-10 b$ during gastrulation (Tingaud-Sequeira et al., 2009; Figure 1A), and therefore their functional relationships need to be elucidated.

\section{REFERENCES}

Alavi, S. M., and Cosson, J. (2006). Sperm motility in fishes. (II) Effects of ions and osmolality: a review. Cell Biol. Int. 30, 1-14.

Armstrong, C. M. (1990). Potassium channel architecture and channel blockers. Prog. Clin. Biol. Res. 334, 1-15.

Barcroft, L. C., Offenberg, H., Thomsen, P., and Watson, A. J. (2003). Aquaporin proteins in murine trophectoderm mediate transepithetion. Dev. Biol. 256, 342-354.

Cerdà, J. (2009). Molecular pathways during marine fish egg hydration: the role of aquaporins. J. Fish Biol. 75, 2175-2196.

Cerdà, J., Fabra, M., and Raldúa, D. (2007). "Physiological and molecular basis of fish oocyte hydration," in The Fish Oocyte: From Basic Studies to Biotechnological Applications, eds lial water movements during cavita-

\section{CONCLUSION AND FUTURE PERSPECTIVES}

Although available information remains scarce, increasing data indicate that aquaporins are involved in water homeostasis in reproductive organs and gametes of teleosts in addition to osmoregulatory mechanisms. At least one paralog (e.g., Aqp 1ab), appears to be tightly regulated both at the gene and protein level during gametogenesis, underlining its importance for the production of gametes well adapted to the reproductive habitats. However, more studies are necessary in teleosts, particularly those using genetic and molecular approaches, as well as specific aquaporin blockers, to elucidate aquaporin function during reproduction. An understanding of the involvement of aquaporins in fluid movement in the teleost gonads and gametes may also lead to improved cryopreservation protocols to assist breeding and species conservation programs.

\section{ACKNOWLEDGMENTS}

Research of the authors was supported by grants from the Spanish Ministry of Science and Innovation and European Commission to Joan Cerdà.

P. J. Babin, J. Cerdà, and E. Lubzens (Dordrecht: Springer), 349-396.

Cerdà, J., and Finn, R. N. (2010). Piscine aquaporins: an overview of recent advances. J. Exp. Zool. A Ecol. Genet. Physiol. 313, 623-650.

Chaube, R., Chauvigné, F., TingaudSequeira, A., Joy, K. P., Acharjee, A., Singh, V., and Cerdà, J. (2011). Molecular and functional characterization of catfish (Heteropneustes fossilis) aquaporin-1b: changes in expression during ovarian development and hormoneinduced follicular maturation. Gen. Comp. Endocrinol. 170 , 162-171.

Chauvigné, F., Lubzens, E., and Cerdà, J. (2011). Design and characterization of genetically engineered zebrafish aquaporin-3 mutants highly permeable to the cryoprotectant ethylene glycol. BMC Biotechnol. 11, 34 . doi:10.1186/1472-6750-11-34 
Chen, L. M., Zhao, J., Musa-Aziz, R., Pelletier, M. F., Drummond, I. A., and Boron, W. F. (2010a). Cloning and characterization of a zebrafish homologue of human AQP1: a bifunctional water and gas channel. Am. J. Physiol. Regul. Integr. Comp. Physiol. 299, R1163-R1174.

Chen, S. X., Bogerd, J., García-López, A., de Jonge, H., de Waal, P. P., Hong, W. S., and Schulz, R. W. (2010b). Molecular cloning and functional characterization of a zebrafish nuclear progesterone receptor. Biol. Reprod. 82, 171-181.

Chen, Q., Peng, H., Lei, L., Zhang, Y., Kuang, H., Cao, Y., Shi, Q. X., Ma, T., and Duan, E. (2011a). Aquaporin 3 is a sperm water channel essential for postcopulatory sperm osmoadaptation and migration. Cell Res. 21, 922-933.

Chen, S. X., Bogerd, J., Andersson, E., Almeida, F. F., Taranger, G. L., and Schulz, R. W. (2011b). Cloning, pharmacological characterization, and expression analysis of Atlantic salmon (Salmo salar L.) nuclear progesterone receptor. Reproduction 141, 491-500.

Cornish, E. J., Hassan, S. M., Martin, J. D., Li, S., and Merzdorf, C. S. (2009). A microarray screen for direct targets of Zicl identifies an aquaporin gene, aqp-3b, expressed in the neural folds. Dev. Dyn. 238, 1179-1194.

Cosson, J., Dreanno, C., Fauvel, C., Groison, A. L., Suquet, M., and Billard, R. (2008). Marine fish spermatozoa: racing ephemeral swimmers. Reproduction 136, 277-294.

Edashige, K., Ohta, S., Tanaka, M., Kuwano, T., Valdez, D. M. Jr., Hara, T., Jin, B., Takahashi, S., Seki, S., Koshimoto, C., and Kasai, M. (2007). The role of aquaporin 3 in the movement of water and cryoprotectants in mouse morulae. Biol. Reprod. 77, 365-375.

Edashige, K., Sakamoto, M., and Kasai, M. (2000). Expression of mRNAs of the aquaporin family in mouse oocytes and embryos. Cryobiology 40, 171-175.

Fabra, M., Raldúa, D., Bozzo, M. G., Deen, P. M., Lubzens, E., and Cerdà, J. (2006). Yolk proteolysis and aquaporin-1o play essential roles to regulate fish oocyte hydration during meiosis resumption. Dev. Biol. 295, 250-262.

Fabra, M., Raldúa, D., Power, D. M., Deen, P. M., and Cerdà, J. (2005). Marine fish egg hydration is aquaporin mediated. Science 307, 545.

Finn, R. N., and Cerdà, J. (2011). Aquaporin evolution in fishes. Front.
Physiol. 2:44. doi:10.3389/fphys. 2011.00044

Finn, R. N., and Fyhn, H. J. (2010). Requirement for amino acids in ontogeny of fish. Aquacult. Res. 41, 684-716.

Finn, R. N., and Kristoffersen, B. A. (2007). Vertebrate vitellogenin gene duplication in relation to the "3R hypothesis": correlation to the pelagic egg and the oceanic radiation of teleosts. PLoS ONE 2, e169. doi:10.1371/journal.pone.0000169

Froger, A., Clemens, D., Kalman, K., Németh-Cahalan, K. L., Schilling, T. F., and Hall, J. E. (2010). Two distinct aquaporin 0s required for development and transparency of the zebrafish lens. Invest. Ophthalmol. Vis. Sci. 51, 6582-6592.

Fyhn, H. J., Finn, R. N., Reith, M., and Norberg, B. (1999). Yolk protein hydrolysis and oocyte free amino acids as key features in the adaptive evolution of teleost fishes to seawater. Sarsia 84, 451-456.

Hanna, R. N., Daly, S. C., Pang, Y., Anglade, I., Kah, O., Thomas, P., and Zhu, Y. (2010). Characterization and expression of the nuclear progestin receptor in zebrafish gonads and brain. Biol. Reprod. 82, 112-122.

Hermo, L., Krzeczunowicz, D., and Ruz, R. (2004). Cell specificity of aquaporins 0,3 , and 10 expressed in the testis, efferent ducts, and epididymis of adult rats. J. Androl. 25, 494-505.

Ikeda, M., Andoo, A., Shimono, M., Takamatsu, N., Taki, A., Muta, K., Matsushita, W., Uechi, T., Matsuzaki, T., Kenmochi, N., Takata, K., Sasaki, S., Ito, K., and Ishibashi, K. (2011). The NPC motif of aquaporin-11, unlike the NPA motif of known aquaporins, is essential for full expression of molecular function. $J$. Biol. Chem. 286, 3342-3350.

Jablonski, E. M., McConnell, N. A., Hughes, F. M. Jr., and Huet-Hudson, Y. M. (2003). Estrogen regulation of aquaporins in the mouse uterus: potential roles in uterine water movement. Biol. Reprod. 69, 1481-1487.

Jacoby, S. C., Gagnon, E., Caron, L., Chang, J., and Isenring, P. (1999). Inhibition of $\mathrm{Na}(+)-\mathrm{K}(+)-2 \mathrm{Cl}(-)$ cotransport by mercury. Am. J. Physiol. 277, C684-C692.

Jin, B., Kawai, Y., Hara, T., Takeda, S., Seki, S., Nakata, Y. I., Matsukawa, K., Koshimoto, C., Kasai, M., and Edashige, K. (2011). Pathway for the movement of water and cryoprotectants in bovine oocytes and embryos. Biol. Reprod. doi:10.1095/biolreprod. 110.088641. [Epub ahead of print].
Kagawa, H., Horiuchi, Y., Kasuga, Y., and Kishi, T. (2009). Oocyte hydration in the Japanese eel (Anguilla japonica) during meiosis resumption and ovulation. J. Exp. Zool. A Ecol. Genet. Physiol. 311A, 752-762.

Kagawa, H., Kishi, T., Gen, K., Kazeto, Y., Tosaka, R., Matsubara, H., Matsubara, T., and Sawaguchi, S. (2011). Expression and localization of aquaporin $1 \mathrm{~b}$ during oocyte development in the Japanese eel (Anguilla japonica). Reprod. Biol. Endocrinol. 9, 71 .

Kristoffersen, B. A., and Finn, R. N. (2008). Major osmolyte changes during oocyte hydration of a clupeocephalan marine benthophil: Atlantic herring (Clupea harengus). Mar. Biol. 154, 683-692.

Kristoffersen, B. A., Nerland, A., Nilsen, F., Kolarevic, J., and Finn, R. N. (2009). Genomic and proteomic analyses reveal nonneofunctionalized vitellogenins in a basal clupeocephalan, the Atlantic herring, and point to the origin of maturational yolk proteolysis in marine teleosts. Mol. Biol. Evol. 26, 1029-1044.

Ma, T., Song, Y., Yang, B., Gillespie, A., Carlson, E. J., Epstein, C. J., and Verkman, A. S. (2000). Nephrogenic diabetes insipidus in mice lacking aquaporin-3 water channels. Proc. Natl. Acad. Sci. U.S.A. 97, 4386-4391.

Marshall, W. S., and Grosell, M. (2006). "Ion transport, osmoregulation and acid-base balance" in The Physiology of Fishes, 3rd Edn, eds D. H. Evans and J. B. Claiborne (Boca Raton: CRC Press), 177-230.

Martinez, A. S., Cutler, C. P., Wilson, G. D., Phillips, C., Hazon, N., and Cramb, G. (2005). Regulation of expression of two aquaporin homologs in the intestine of the European eel: effects of seawater acclimation and cortisol treatment. Am. J. Physiol. Regul. Integr. Comp. Physiol. 288, R1733-R1743.

Miura, T., Higuchi, M., Ozaki, Y., Ohta, T., and Miura, C. (2006). Progestin is an essential factor for the initiation of the meiosis in spermatogenetic cells of the eel. Proc. Natl. Acad. Sci. U.S.A. 103, 7333-7338.

Monzani, E., Bazzotti, R., Perego, C., and La Porta, C. A. (2009). AQP1 is not only a water channel: it contributes to cell migration through Lin7/betacatenin. PLoS ONE 4, e6167. doi:10.1371/journal.pone.0006167

Morishita, Y., Matsuzaki, T., Harachikuma, M., Andoo, A., Shimono, M., Matsuki, A., Kobayashi, K., Ikeda, M., Yamamoto, T., Verkman,
A., Kusano, E., Ookawara, S., Takata, K., Sasaki, S., and Ishibashi, K. (2005). Disruption of aquaporin-11 produces polycystic kidneys following vacuolization of the proximal tubule. Mol. Cell Biol. 25, 7770-7779.

Oliveira, C. A., Carnes, K., França, L. R., Hermo, L., and Hess, R. A. (2005). Aquaporin-1 and -9 are differentially regulated by oestrogen in the efferent ductule epithelium and initial segment of the epididymis. Biol. Cell 97, 385-395.

Papadopoulos, M. C., Saadoun, S., and Verkman, A. S. (2008). Aquaporins and cell migration. Pflugers Arch. 456, 693-700.

Raldúa, D., Otero, D., Fabra, M., and Cerdà, J. (2008). Differential localization and regulation of two aquaporin-1 homologs in the intestinal epithelia of the marine teleost Sparus aurata. Am. J. Physiol. Regul. Integr. Comp. Physiol. 294, R993-R1003.

Santos, C. R., Estêvão, M. D., Fuentes, J., Cardoso, J. C., Fabra, M., Passos, A. L., Detmers, F. J., Deen, P. M., Cerdà, J., and Power, D. M. (2004). Isolation of a novel aquaglyceroporin from a marine teleost (Sparus auratus): function and tissue distribution. $J$. Exp. Biol. 207, 1217-1227.

Schulz, R. W., de França, L. R., Lareyre, J. J., Le Gac, F., ChiariniGarcia, H., Nóbrega, R. H., and Miura, T. (2010). Spermatogenesis in fish. Gen. Comp. Endocrinol. 165, 390-411.

Scott, A. P., Sumpter, J. P., and Stacey, N. (2010). The role of the maturation-inducing steroid, 17,20beta-dihydroxypregn-4-en-3one, in male fishes: a review. J. Fish Biol. 76, 183-224.

Singh, V., and Joy, K. P. (2010). An involvement of vasotocin in oocyte hydration in the catfish Heteropneustes fossilis: a comparison with effects of isotocin and hCG. Gen. Comp. Endocrinol. 166, 504-512.

Skowronski, M. T., Kwon, T. H., and Nielsen, S. (2009). Immunolocalization of aquaporin 1,5 , and 9 in the female pig reproductive system. $J$. Histochem. Cytochem. 57, 61-67.

Sohara, E., Ueda, O., Tachibe, T., Hani, T., Jishage, K., Rai, T., Sasaki, S., and Uchida, S. (2007). Morphologic and functional analysis of sperm and testes in aquaporin 7 knockout mice. Fertil. Steril. 87, 671-676.

Sumanas, S., Jorniak, T., and Lin, S. (2005). Identification of novel vascular endothelial-specific genes by the microarray analysis of the zebrafish cloche mutants. Blood 106, 534-541. 
Thomas, P., Pang, Y., Zhu, Y., Detweiler, C., and Doughty, K. (2004). Multiple rapid progestin actions and progestin membrane receptor subtypes in fish. Steroids 69, 567-573.

Thoroddsen, A., Dahm-Kähler, P., Lind, A. K., Weijdegård, B., Lindenthal, B., Müller, J., and Brännström, M. (2011). The water permeability channels aquaporins 1-4 are differentially expressed in granulosa and theca cells of the preovulatory follicle during precise stages of human ovulation. J. Clin. Endocrinol. Metab. 96, 1021-1028.

Tingaud-Sequeira, A., Calusinska, M., Finn, R. N., Chauvigné, F., Lozano, J., and Cerdà, J. (2010). The zebrafish genome encodes the largest vertebrate repertoire of functional aquaporins with dual paralogy and substrate specificities similar to mammals. BMC Evol. Biol. 10, 38. doi:10.1186/1471-2148-10-38

Tingaud-Sequeira, A., Chauvigné, F., Fabra, M., Lozano, J., Raldúa, D., and Cerdà, J. (2008). Structural and functional divergence of two fish aquaporin-1 water channels following teleost-specific gene duplication. BMC Evol. Biol. 8, 259. doi:10.1186/1471-2148-8-259

Tingaud-Sequeira, A., Zapater, C., Chauvigné, F., Otero, D., and Cerdà, J. (2009). Adaptive plasticity of killifish (Fundulus heteroclitus) embryos: dehydrationstimulated development and differential aquaporin-3 expression. Am. J. Physiol. Regul. Integr. Comp. Physiol. 296, R1041-R1052.

Tubbs, C., Tan, W., Shi, B., and Thomas, P. (2011). Identification of 17,20ß,21-trihydroxy-4-pregnen3 -one $(20 \beta-S)$ receptor binding and membrane progestin receptor alpha on southern flounder sperm (Paralichthys lethostigma) and their likely role in 20(B-S stimulation of sperm hypermotility. Gen. Comp. Endocrinol. 170, 629-639.

Yang, B., Song, Y., Zhao, D., and Verkman, A. S. (2005). Phenotype analysis of aquaporin-8 null mice. Am. J. Physiol. Cell Physiol. 288, C1161C1170.

Yeung, C. H. (2010). Aquaporins in spermatozoa and testicular germ cells: identification and potential role. Asian J. Androl. 12, 490-499.

Yeung, C. H., Callies, C., Rojek, A., Nielsen, S., and Cooper, T. G. (2009). Aquaporin isoforms involved in physiological volume regulation of murine spermatozoa. Biol. Reprod. 80, 350-357.

Zapater, C., Chauvigné, F., Norberg, B., Finn, R. N., and Cerdà, J. (2011). Dual neofunctionalization of a rapidly evolving aquaporin1 paralog resulted in constrained and relaxed traits controlling channel function during meiosis resumption in teleosts. Mol. Biol. Evol. doi: $10.1093 / \mathrm{molbev} / \mathrm{msr} 146$. [Epub ahead of print].

Zilli, L., Schiavone, R., Chauvigné, F., Cerdà, J., Storelli, C., and Vilella, S. (2009). Evidence for the involvement of aquaporins in sperm motility activation of the teleost gilthead sea bream (Sparus aurata). Biol. Reprod. 81, 880-888.

Zilli, L., Schiavone, R., Storelli, C. and Vilella, S. (2008). Molecular mechanisms determining sperm motility initiation in two sparids (Sparus aurata and Lithognathus mormyrus). Biol. Reprod. 79, 356-366.

Conflict of Interest Statement: The authors declare that the research was conducted in the absence of any commercial or financial relationships that could be construed as a potential conflict of interest.

Received: 24 May 2011; accepted: 13 September 2011; published online: 30 September 2011.

Citation: Chauvigné F, Zapater C and Cerdà J (2011) Role of aquaporins during teleost gametogenesis and early embryogenesis. Front. Physio. 2:66. doi: 10.3389/fphys.2011.00066

This article was submitted to Frontiers in Aquatic Physiology, a specialty of Frontiers in Physiology.

Copyright (c) 2011 Chauvigné, Zapater and Cerdà. This is an open-access article subject to a non-exclusive license between the authors and Frontiers Media $S A$, which permits use, distribution and reproduction in other forums, provided the original authors and source are credited and other Frontiers conditions are complied with. 